\title{
Acute psychosis in two elderly patients
}

\author{
Kathryn E Anderson, John M Starr, Richard I Lindley
}

Case 1

A 96-year-old woman, residing in a nursing home, presented with acute paranoid ideation of sudden onset. Her carers reported that, although normally lucid and mentally alert, she had suddenly decided that they were ignoring her and was convinced that they were on strike. At lunchtime she refused to eat, claiming her meal was poisoned. Her niece visited that day and commented that she felt her aunt's eyesight did not appear as good as usual. There were no other physical symptoms of note and her state of belief lasted several days. Her medical history consisted of migraine, irritable bowel syndrome, hypertension, and depression diagnosed following the death of her husband. She had no other psychiatric history. Her medication consisted of mebeverine $135 \mathrm{mg}$ tid, amitryptiline $25 \mathrm{mg}$, bendrofluazide $2.5 \mathrm{mg}$, and pizotifen $0.5 \mathrm{mg}$. She was teetotal and a lifelong non-smoker.

A visit 5 days after presentation by a consultant psychiatrist found her still paranoid with difficulty registering information. Some 13 days later she was somewhat improved and when a consultant physician visited, she commented that her ideas had been abnormal. Physical examination confirmed hypertension (blood pressure $190 / 105 \mathrm{mmHg}$ ) and a large abdominal aortic aneurysm. Routine investigations including infection screening were unremarkable. Her computed tomography (CT) scan is pictured here (figure 1).

\section{Case 2}

A 78-year-old woman was admitted to the psychiatric unit with a history of sudden perplexion and confusion. She normally cared for her grandson and suddenly felt acutely unable to do so. She also felt paranoid and was convinced that her family were lying to her. On admission she described a feeling of altered sensation in her right hand which felt 'numb'. Her medical history included depression and agoraphobia and she had a previous admission with an acute psychosis 3 years previously, diagnosed as agitated depression. Her medication consisted of haloperidol $0.5 \mathrm{mg}$ daily. She smoked 10 cigarettes a day and drank 15 units of alcohol a week. On examination she was agitated and scored $21 / 30$ on a mini-mental state examination. She improved without any intervention. She was reviewed by a physician 10 days after admission and was found to be alert and orientated; she denied any of her previously held beliefs. On examination she was noted to have reduced fine finger movements of her right hand and increased lower limb reflexes, particularly on the right. Her plantar responses were both flexor. All routine investigations were unremarkable. Her CT brain scan is shown in figure 2 .

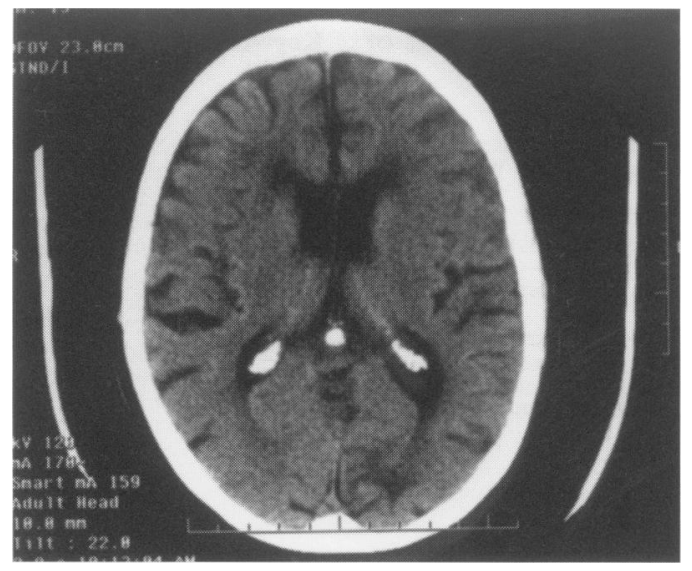

Figure 1 Brain CT scan of case 1

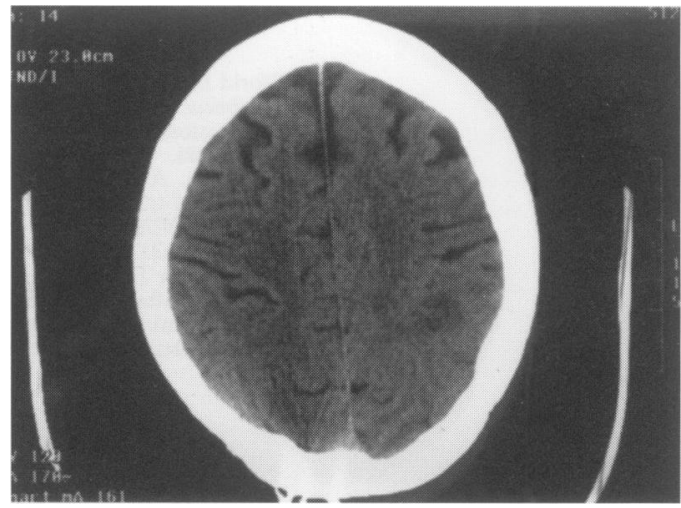

Figure 2 Brain CT scan of case 2 (a)

Victoria Hospital,

Cranleith Road,

Edinburgh EH4 2DN,

Scotland, UK

K E Anderson

J M Starr

R I Lindley

Accepted 10 March 1998
1 What abnormalities are seen on the CT scans?

2 What is the diagnosis? 


\section{Answers}

QUESTION 1

Figure 1 shows an occipital infarct. Figure 2 shows a left subcortical infarct.

QUESTION 2

Acute psychosis presenting as new cerebral infarction.

\section{Discussion}

Acute psychotic episodes in the elderly are not unusual, particularly when associated with the commonly recognised acute confusional state of any organic illness. Psychosis is rarely reported in the literature as a finding secondary to acute cerebral infarction; this may be due to non-recognition.

In both the cases described, the physical symptoms, ie, altered vision (case 1), numbness of right hand followed by right-sided neurological signs (case 2), were newly reported and correlate with the areas of infarction seen on CT scanning. Alterations of physical state are readily recognised as common sequelae of stroke. Stroke is defined as "focal or global neurological impairment of sudden onset lasting longer than 24 hours (or leading to death) and of presumed vascular aetiology". ${ }^{1}$ The term neurological impairment includes not only physical deficits but also applies to impairments of mental state. This presents commonly as impairment of cognitive function, but may also include altered perception, such as psychosis.

Stroke is well recognised as a major cause of cognitive impairment and dementia, ${ }^{2-4}$ and impairment of mood and control of emotional response, eg, apathy, anxiety and pathological emotional lability, are also well documented following stroke. ${ }^{5}$ Depression in particular has been linked with stroke. One recent study highlighted the prevalence of 'silent' cerebral infarction in patients with major depression. ${ }^{6}$ This can be compared with the model of schizophrenia in which psychotic symptoms of

1 World Health Organisation (WHO). Cerebrovascular disease. A clinical and research classification. Geneva: WHO Offset publication, 1978; p 43.

2 Hachinski VC, Lassen NA, Marshall J. Multi-infarct dementia. A cause of mental deterioration in the elderly. dementia. A cause

3 Lancet $1974 ; 2: 207-9$. Vascular dementia: fortnightly review. Amer K, Wilcock G. Vasc

BMF 1996;312:227-31.

3 months after stroke. Baseline frequency and effect of dif 3 months after stroke. Baseline frequency and effect of different definitions of dementia in the Helsinki stroke a
memory study (SAM) cohort. Stroke 1997;28:785-92.

\section{Summary points}

acute psychotic episodes may follow cerebra infarction

- patients with a previous psychiatric history may be at particular risk

- acute cerebral infarction should be considered in the differential diagnosis of the elderly patient presenting with acute psychosis or depression

- a detailed neurological examination and evaluation of stroke risk factors should form part of the diagnostic process

the illness formerly attributed to a 'functional aetiology' are now similarly related to structural brain abnormalities such as cerebral atrophy and ventricular enlargement. ${ }^{8}$

Half of the few previously reported episodes of stroke-induced psychosis have occurred in patients with a history or family history of mood disorder, ${ }^{5}$ as in both of our patients. Patients with a history of psychiatric disorder may be more prone to psychosis following stroke and any new episodes may be considered as part of their long-standing disorder, rather than any other pathology such as acute stroke.

We suggest that elderly patients presenting with acute psychosis should have particular attention paid to stroke risk factors (eg, hypertension, smoking, diabetes), and receive detailed neurological examination. CT scanning is helpful to confirm the diagnosis; secondary prevention with aspirin and control of risk factors should also be considered. These interventions are likely to reduce risk of further strokes but their effects on prevention of further psychotic episodes are uncertain.

\section{Final diagnosis}

Acute psychosis presenting as new cerebral infarction.

Keywords: stroke; psychosis; mood disorder; elderly

5 Ghika-Schmid F, Bogousslavsky J. Affective disorders following stroke, Eur Neurol 1997;38:75-81.

6 Fulikawa T, Yanai I, Yamawaki S. Psychosocial stressors in patients with major depression and silent cerebral infarction. Stroke 1997;4:1123-5.

7 Davison K, Bagley CR. Schizophrenia-like psychosis associated with organic disorders of the CNS. A review of the literature. Curr Prob Neuropsychiatry 1969;113-62.

8 Lewis S. Brian imaging in psychiatry: another look. $\mathrm{Br} \mathcal{f}$ Hosp Med 1992; 47:175-83. 